\title{
Endoscopic aspiration mucosectomy using a novel vibration hood
}

We recently reported the usefulness of three types of original prelooped caps: the irrigating prelooped cap [1], the twochannel prelooped hood [2], and the internally retained snare hood [3] for endoscopic aspiration mucosectomy (EAM). EAM is the simplest method of endoscopic mucosal resection (EMR) [1 -5], but there is a risk of aspirating and perforating the full thickness of the gastric wall. To reduce the risk of such perforation, the resection is normally performed by strangulation with a snare using a light touch. Experience is needed to apply this light touch with just the right pressure, and it can be an uncertain process. In order to apply vibration during strangulation, a novel vibration hood has been developed that enables strangulation and resection of only the mucosal and submucosal layers, by vibrating the snare during strangulation to shake off the muscle layer and serous membrane ( $\bullet$ Fig. 1 ).

A commercial tip hood (Create Medic Co., Ltd., Yokohama, Japan) was modified ( $\bullet$ Fig. 2). First, a side hole was made in the hood ( $\bullet$ Fig. 2 b), e) so that the Lshaped oscillating part (g) did not transmit vibrations outside the vibrating portion. In the L-shaped oscillating part (g), there is a forceps aperture $(\mathrm{h})$, made so as to maintain straight concentricity with the forceps tube (i) of the hood (e). The Lshaped oscillator ( $g$ ) is fixed to an eccentric motor (b) (Shicoh Co., Ltd, Japan) ( $\mathrm{Fig.3}$ ), which produces the vibration. To prevent the vibration of the vibrating part (consisting of $b+g$ ) from being transmitted to the other parts, including the endoscope body (a), hood (e), and cap section, a vibration absorption section consisting of a vibration attenuator (c) made from urethane impact absorption material is sealed in the vibrating portion. The vibration absorption section is fixed to the cap (f). With this construction, the vibration is transmitted only to the treatment tool inserted into both the forceps tube (i) of the hood (e) and the forceps aperture in the oscillating section (h). Thus, an endoscopic procedure with vibration is possible. Power is supplied from the main power source via the connector, and variable vibration frequencies are possible ( Fig.4a). Vibration is ob-

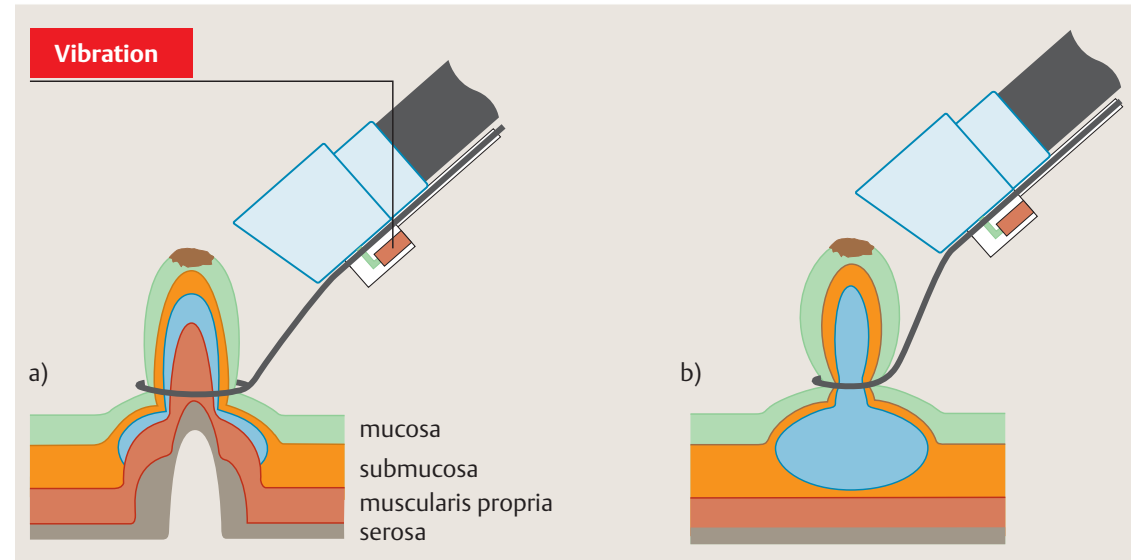

Fig. 1 Vibratory effect for endoscopic aspiration mucosectomy.

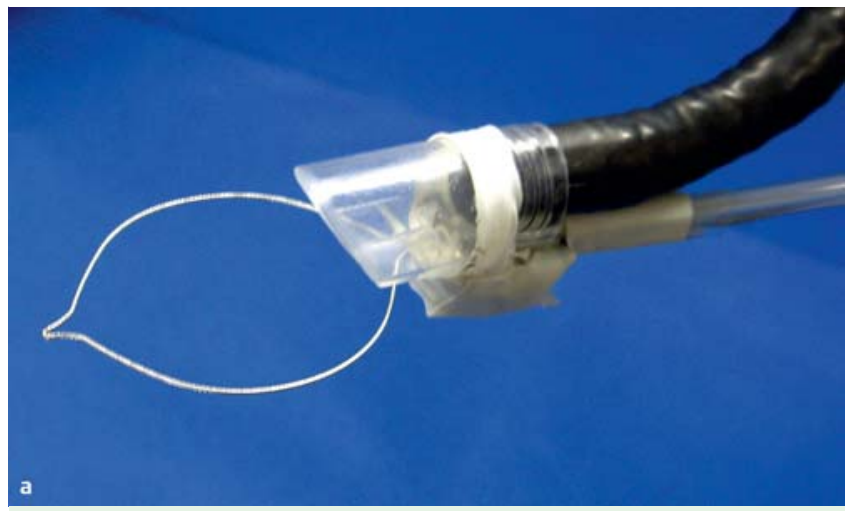

Fig. 2 a Vibration hood at the tip of the endoscope. b Diagrammatic cross-sections of the vibration hood.

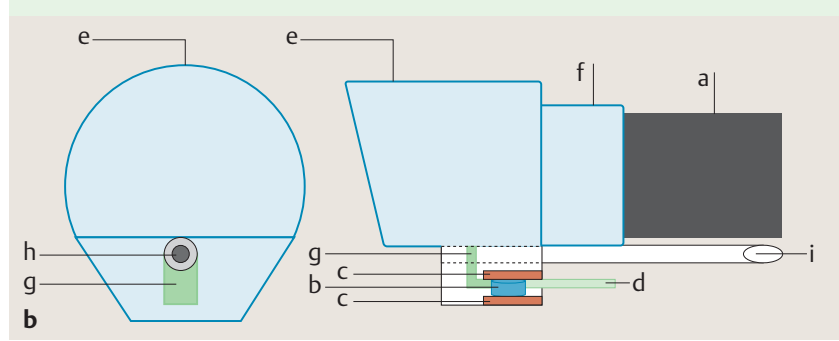

tained by stepping on a foot switch connected to the power source of the device (॰ Fig. 4b).

The first trial EAM was conducted using a resected pig stomach. Five milliliters of physiological saline were injected locally into the submucosa. The central portion of the protrusion was then aspirated and resected by strangulating with the snare. In a following study, investigations were conducted separately in 18 animal lesions with and without vibration at $10000 \mathrm{rpm}$ applied at the time of strangulation and resection. Six resected porcine stomachs were used. Each resected porcine stomach was subjected to EAM systematically at three locations ( Fig.5). Nine EAMs were done with each vibration frequency ( $0 \mathrm{rpm}$ and $10000 \mathrm{rpm}$ ), giving a total of 18 EAMs. The vibration load was evenly divided so that bias would not occur due to differences in location ( $\bullet$ Table 1 ).

EAM was simpler when using the novel vibration hood than when the vibration hood was not used. Two perforations occurred, and these were in the no-vibration group ( $\bullet$ Table 1 ). Perforation rates were lower in the vibration group 


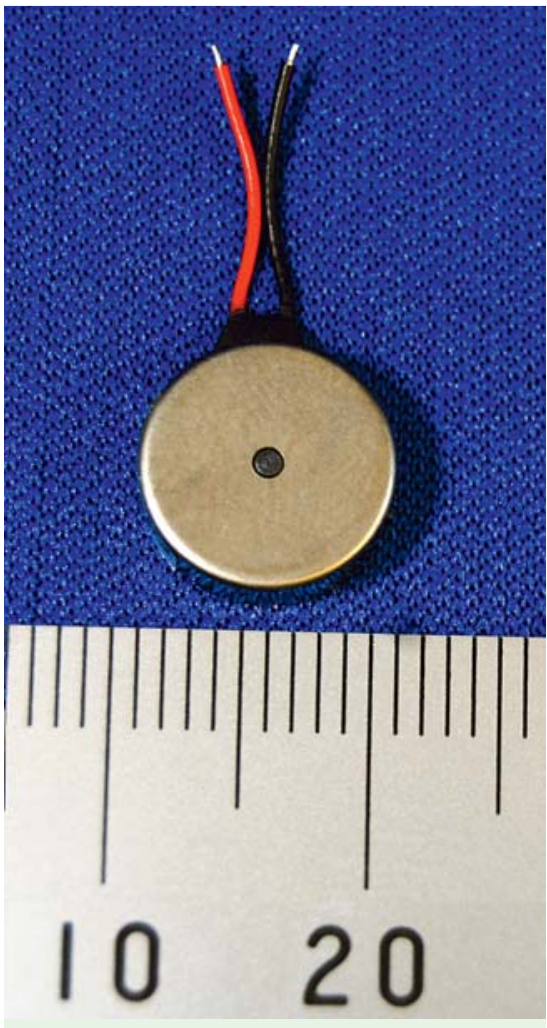

Fig. 3 Vibration motor

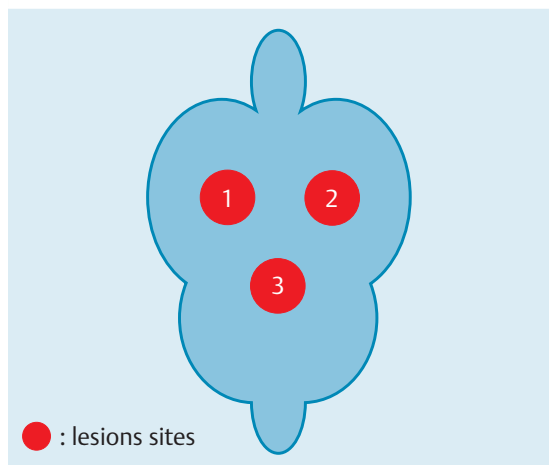

Fig. 5 The three locations of experimental EAM in resected pig stomachs.

$(0 / 9: 0 \%)$ than in the no-vibration group (2/9:22.2\%).

In this study, I attempted to increase the safety of EAM by vibrating the snare during strangulation. I have reported that

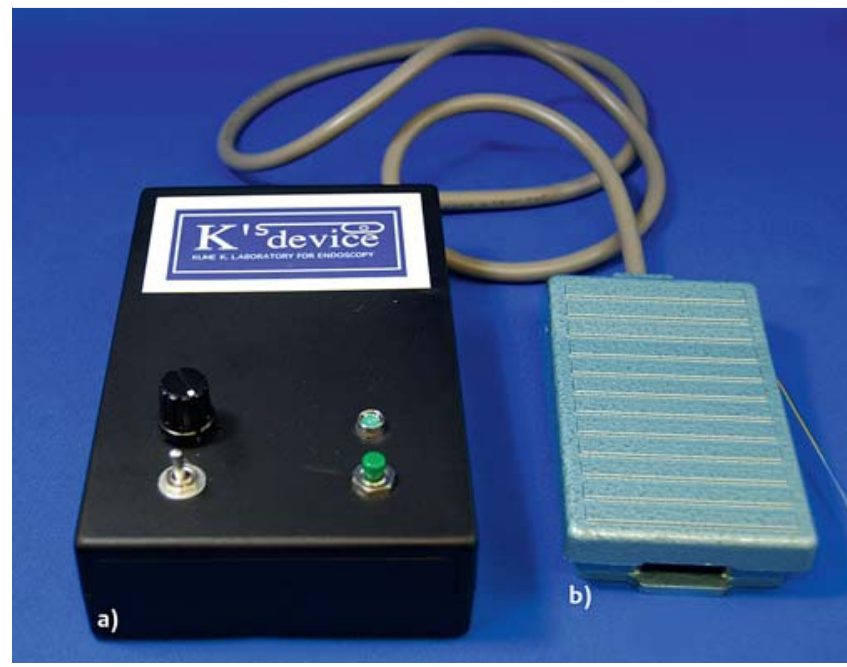

Fig. 4 Variable vibration frequencies are supplied from the device box (a) and a foot switch (b).

when performing peripheral incisions and submucosal dissection with a knife in $E S D$, the time for the procedure was reduced by adding vibration [6]. When EAM was performed using this hood, vibration was applied from the start of the strangulation with the snare until the resection was completed. Stepping on a foot switch was all that was needed to apply the vibration load, so an experienced light touch was not necessary.

This hood is equipped with a vibration attenuator, and though not $100 \%$ of the vibration was prevented from being transmitted to the endoscope hood, the image was not distorted or blurred. The eccentric motor vibrates at a frequency of $10000 \mathrm{rpm}$.

To gain universal acceptance of the procedure of EMR with aspiration, it is crucial to make the strangulating and resecting process universally applicable. Safe EAM will become possible when even inexperienced endoscopic surgeons can perform strangulation and resection easily and safely. In the author's previous investigations there has been a high rate of enbloc resection of lesions less than $15 \mathrm{~mm}$ in size with EAM, and this size is thought to be appropriate for EAM using this hood [1-3].
In conclusion, EAM using a novel vibration hood seems to be useful in reduction of the risk of perforation.

\section{Acknowledgment}

$\nabla$

This work was supported by Grant-in-Aid for Scientific Research (MEXT KAKENHI: 19590742).

Endoscopy_UCTN_Code_TTT_1AO_2AG

\section{K. Kume}

Third Department of Internal Medicine, University of Occupational and Environmental Health, Japan, School of Medicine, Kitakyusyu, Japan

\begin{tabular}{llll} 
Resected pig stomach & Lesion $\mathbf{1}$ & Lesion 2 & Lesion 3 \\
1 & - & + & - \\
\hline 2 & + & $-(\mathrm{P})$ & + \\
\hline 3 & - & + & + \\
\hline 4 & + & - & - \\
\hline 5 & $-(\mathrm{P})$ & - & + \\
\hline
\end{tabular}

+, with vibration; -, without vibration; (P), perforation.
Table 1 Locations and modes (with or without vibration) of experimental endoscopic aspiration mucosectomy in six resected pig stomachs. 


\section{References}

1 Kume K, Yamasaki M, Kubo $K$ et al. EMR of upper GI lesions when using a novel soft, irrigation, prelooped hood. Gastrointest Endosc 2004; 60: 124-128

2 Kume K, Yamasaki M, Kanda Ket al. Grasping forceps-assisted endoscopic mucosal resection of early gastric cancer with a novel 2channel prelooped hood. Gastrointest Endosc 2006; 64: 108-112

3 Kume K, Yamasaki M, Tashiro M et al. Endoscopic mucosal resection for early gastric cancer: comparison of two modifications of the cap method. Endoscopy 2008; 40: 280 283
4 Inoue H, Takeshita K, Hori H et al. Endoscopic mucosal resection with a cap-fitted panendoscope for esophagus, stomach and colon mucosal lesions. Gastrointest Endosc 1993; 39: $58-62$

5 Torii A, Sakai M, Kajiyama $M$ et al. Endoscopic aspiration mucosectomy as curative endoscopic surgery: analysis of 24 cases of early gastric cancer. Gastrointest Endosc 1995; 42: 475-479

6 Kume K. Endoscopic submucosal dissection using a novel vibration endoscopy. HepatoGastroenterology (in press)

\section{Bibliography}

DOI $10.1055 / \mathrm{s}-0029-1215023$

Endoscopy 2009; 41: E296 -E298

(c) Georg Thieme Verlag KG Stuttgart · New York . ISSN 0013-726X

\section{Corresponding author}

\section{K. Kume, MD, PhD}

K's device, Kume K. Laboratory for Endoscopy Third Department of Internal Medicine

University of Occupational and Environmental Health, Japan

School of Medicine

1-1, Iseigaoka, Yahatanishi-ku

Kitakyusyu 807-8555

Japan

Fax: +81-93-6920107

k-kume@med.uoeh-u.ac.jp 